\title{
BOUNDS ON CERTAIN NONLINEAR DISCRETE INEQUALITIES
}

\author{
WEIHONG SHENG AND WEI NIAN LI
}

Abstract. Our aim in this paper is to investigate certain nonlinear discrete inequalities which provide explicit bounds on unknown functions. The inequalities given here can be used as tools in applications in the theory of certain finite difference and sum-difference equations.

Mathematics subject classification (2000): 26D15, 39A99.

Key words and phrases: Discrete inequality, bound, nonlinear, difference equation.

\section{REFERENCES}

[1] R. P. Agarwal, Difference equations and inequalities, Marcel Dekker Inc., New York, 1997.

[2] B. G. PACHPATTE, Inequalities for certain finite difference and sum-difference equations, Math. Ineq. Appl. 4(2001), 499-506.

[3] B. G. PACHPATtE, On some fundamental finite difference inequalities, Tamkang J. Math. 32(2001), 217-223.

[4] E. H. YANG, A new nonlinear discrete inequality and its application, Ann. Differential Equations 17(2001), 261-267.

[5] F. W. MENG AND W. N. LI, On some new nonlinear discrete inequalities and their applications, J. Comput. Appl. Math. 158(2003), 407-417.

6] B. G. PACHPATTE, Inequalities for Finite Difference Equations, Marcel Dekker Inc., New York, 2002.

[7] B. G. PACHPATtE, Integral and Finite Difference Inequalities and Applications, Elsevier Science B. V., Amsterdam, 2006.

[8] D. S. Mitrinović, Analytic Inequalities, Springer-Verlag, New York, 1970. 